\title{
Degradation Products of Lambda-Cyhalothrin in Aqueous Solution as Determined by SBSE-GC-IT-MS
}

\author{
Renata Colombo, *,a Janete H. Yariwake ${ }^{b}$ and Marcos R. V. Lanza ${ }^{b}$ \\ ${ }^{a}$ Escola de Artes, Ciências e Humanidades, Universidade de São Paulo, \\ 03828-000 São Paulo-SP, Brazil \\ ${ }^{b}$ Instituto de Química de São Carlos, Universidade de São Paulo, Caixa Postal 780, \\ 13560-970 São Carlos-SP, Brazil
}

\begin{abstract}
Lambda-cyhalothrin is a widely employed pyrethroid insecticide and is potentially toxic to the environment and to human health. Several reports indicate the presence of this insecticide in water samples worldwide, but more data about its behavior in waters and its derivative products are required. This study shows the behavior of the pesticide lambda-cyhalothrin in aqueous solution at different $\mathrm{pH}$ and temperature that were simulated by using water contaminated with a toxic concentration of lambda-cyhalothrin and chemical oxidative process. Lambda-cyhalothrin and its derivative products were monitored employing stir bar sorptive extraction (SBSE), using liquid and thermal desorption, combined with gas chromatography coupled with ion trap mass spectrometry (GC-IT-MS) technology. GC-IT-MS enabled the proposal of the lambda-cyhalothrin chemical transformation pathway and the full identification of twelve derivative products. Among them, only 3-phenoxybenzaldehyde is reported as a degradation product of lambda-cyhalothrin, and this compound is an environmental pollutant with endocrine disrupting activity.
\end{abstract}

Keywords: lambda-cyhalothrin, water, chemical oxidative process, SBSE, GC-IT-MS

\section{Introduction}

Pyrethroids are a class of compounds extensively utilized in agriculture to control insect pests in a wide variety of crops, and in non-agricultural settings such as in public health, gardens and insect pest control of pets. The use of pyrethroid insecticides has increased in recent decades owing to the ban of several organophosphate compounds. ${ }^{1,2}$ Lambda-cyhalothrin is a pyrethroid contained in several brand name products; ${ }^{2}$ its residues have been detected in urban and rural runoff waters worldwide, presenting high potential for bioconcentration,,$^{3-6}$ resulting in toxicity in humans and aquatic organisms. ${ }^{6-12}$ The U. S. Environmental Protection Agency (USEPA) is reviewing the human health and ecological risk assessment from exposure to this compound and the EPA's registration review decision about this compound is still pending. ${ }^{13}$

Despite the reports of the presence of lambda-cyhalothrin in water and sediments of different environments in various countries, ${ }^{14-17}$ proving its environmental toxicity, data about the behavior of lambda-cyhalothrin and its derivative products in water are still insufficient. Lambdacyhalothrin derivative products provided by photolysis and hydrolysis processes were already studied, ${ }^{3,18,19}$ but it is necessary to research the interactions of lambdacyhalothrin under other environmental conditions, such as $\mathrm{pH}$ variation and presence of free radicals. Moreover, quick and easy analytical methods to detect this pollutant and its derivative products in environmental matrices are also scarce. In this work, different environmental conditions were used to study lambda-cyhalothrin behavior and its derivative products in water, including the identification of eleven products not yet described as degradation products of lambda-cyhalothrin. Analytical methods were developed based on stir bar sorptive extraction (SBSE), using liquid and thermal desorption in combination with gas chromatography (GC) coupled with ion trap mass spectrometry (IT-MS). To our knowledge this is also the first report that combines the solvent-free, environmentally friendly thermal desorption techniques (SBSE and GC) to study lambda-cyhalothrin and its degradation products.

*e-mail: renatacolomb@gmail.com 


\section{Experimental}

\section{Reference substances, chemicals, and reagents}

All analytical standards were obtained from Sigma-Aldrich: lambda-cyhalothrin (97\% purity) and 3-phenoxybenzaldehyde (98\% purity). All the other chemicals were analytical or HPLC grade from Mallinckrodt, Merck and Ecibra. Purified water (resistivity $18.2 \mathrm{M} \Omega \mathrm{cm}$ ) was obtained using a Milli-Q water purification system.

\section{Lambda-cyhalothrin assays in aqueous and oxidant medium}

The behavior of lambda-cyhalothrin in aqueous and oxidant medium was evaluated using a stock solution of lambda-cyhalothrin at $4.5 \mu \mathrm{g} \mathrm{L}^{-1}$ (level in water or sediment that has shown toxic effects on aquatic organisms). The univariate assays were performed in a recirculation flow-through reactor, coupled to a thermostatically controlled bath at $25^{\circ} \mathrm{C}$, using different levels of $\mathrm{pH}$ (1.5 and 4.0, both adjusted with hydrochloric acid; 6.2, original pH; 8.0, 10.0 and 11.0 , adjusted by using a $0.01 \mathrm{~mol} \mathrm{~L}^{-1}$ sodium hydroxide solution) and different concentrations of hydrogen peroxide $\left(5,10,20,50,100,340,680\right.$ and $\left.1700 \mu g \mathrm{~L}^{-1}\right)$. The range for the hydrogen peroxide concentration was determined considering the stoichiometry of the complete oxidation of lambda-cyhalothrin at $4.5 \mu \mathrm{g} \mathrm{L}^{-1}$ (1 mol of lambdacyhalothrin $=5.5 \mathrm{~mol}$ of $\mathrm{H}_{2} \mathrm{O}_{2}$ ).

The reaction mixtures were magnetically stirred for $24 \mathrm{~h}$ and sample aliquots of the reaction mixture were collected at predetermined reaction times $(0,1,2,4,6,16,20,22$, and $24 \mathrm{~h}$ ). The derivative experiments were performed in triplicate to confirm the repeatability of the measurements.

\section{SBSE extraction and desorption}

The products of the chemical oxidation process were extracted using SBSE stir bars Twister ${ }^{\circledR}$ (Gerstel) with polydimethylsiloxane $(\mathrm{PDMS})$ coating $\left(10 \times 0.5 \mathrm{~mm}^{2}\right.$, $24 \mu \mathrm{L}$ PDMS), stirred at $700 \mathrm{rpm}$.

The extraction conditions were optimized using a $2^{5-2}$ fractional factorial design created with the help of Minitab ${ }^{\circledR}$ version 13 software $^{20}$ (Minitab Inc, USA). The fractional factorial design was composed of the 11 experiments (runs) including 1 replicate (central point). The variables examined were the percentage of organic modifier (methanol), variation of ionic strength (addition of $\mathrm{NaCl}$ ), $\mathrm{pH}$, temperature and extraction time. Each variable was considered as a factor in the experimental model, and the two extreme levels were attributed to each of these factors (15-40\% of methanol; $0.5-12 \%$ of $\mathrm{NaCl}$; $\mathrm{pH} 1.50-10.00$; temperature $=25-60{ }^{\circ} \mathrm{C}$ and $30-120$ min of extraction time). The maximum and minimum levels were defined based on preliminary experiments. After the extraction procedure, the stir bar was washed with ultrapure water and dried with a lint-free tissue prior to the liquid desorption of the analytes with $1 \mathrm{~mL}$ of methanol for $120 \mathrm{~min}$.

The desorption of lambda-cyhalothrin derivative products from the SBSE stir bar was optimized by comparing liquid and thermal processes. Liquid desorption was optimized inserting the stir bar into a vial containing $1 \mathrm{~mL}$ of solvent (methanol, ethanol or acetonitrile) and sonicated at room temperature $\left(25^{\circ} \mathrm{C}\right)$ for different lengths of time $(15,30,60,90$ or $120 \mathrm{~min})$. In thermal desorption, different injector temperature programs were evaluated to define the most efficient desorption temperature of the analytes from the stir bar.

\section{GC-IT-MS analysis}

The GC-IT-MS analysis was performed in a CP 3800 gas chromatograph equipped with a ChromatoProbe ${ }^{\circledR}$ device coupled to a GC-IT-MS/MS Saturn 2000 (Varian). The GC analyses were performed using a DB-5 ms fused silica capillary column $(30 \mathrm{~m} \times 0.25 \mathrm{~mm}$ i.d., $0.5 \mu \mathrm{m}$ film thickness, Agilent) and helium as carrier gas at a flow rate of $1.2 \mathrm{~mL} \mathrm{~min}^{-1}$. The oven temperature was programmed from $75^{\circ} \mathrm{C}$ (held for $1 \mathrm{~min}$ ) to $275^{\circ} \mathrm{C}$ (held for $7 \mathrm{~min}$ ), at a heating rate of $20{ }^{\circ} \mathrm{C} \mathrm{min}{ }^{-1}$. The injector temperature program was $45^{\circ} \mathrm{C}$ (held for $1 \mathrm{~min}$ ) to $255^{\circ} \mathrm{C}$ (held for $12 \mathrm{~min}$ ), at a heating rate of $42{ }^{\circ} \mathrm{C} \mathrm{min}^{-1}$. The IT-MS analyses were performed in scan mode $(\mathrm{m} / \mathrm{z}, 40$ to 450$)$, using electron impact ionization $(70 \mathrm{eV})$ in positive mode. The temperature of the transfer line, ion trap and manifold were set at 300,220 and $40{ }^{\circ} \mathrm{C}$, respectively.

\section{Results and Discussion}

\section{Lambda-cyhalothrin behavior in oxidative medium}

In oxidizing and acidic medium ( $\mathrm{pH} 1.5$ and 4.0), after $24 \mathrm{~h}$, no transformation in the lambda-cyhalothrin molecule or derivative products were detected. Under these conditions, the lambda-cyhalothrin solution become a particulate suspension during stirring. This physical transformation justifies and corroborates reported studies showing that at $\mathrm{pH}<4$ the adsorption of lambda-cyhalothrin in soil and sediments is slightly higher than at $\mathrm{pH} 9 .^{3,8,16,21} \mathrm{On}$ the other hand, lambda-cyhalothrin was not inert in aqueous solution ( $\mathrm{pH}$ 6.2) and alkaline $(\mathrm{pH} 8.0,10.0$ and 11.0) medium; under the tested conditions it was transformed into several intermediate and final derivative products. The 
conditions in which lambda-cyhalothrin showed the highest number of derivative reaction products (twelve compounds, in larger amount than in other reaction times) in this study was $4.5 \mu \mathrm{g} \mathrm{L}^{-1}$ of lambda-cyhalothrin, $680 \mu \mathrm{g} \mathrm{L}^{-1}$ of hydrogen peroxide, and $4 \mathrm{~h}$ of reaction time at $\mathrm{pH} 10.0$. Since simple, rapid methodologies for simultaneous analysis of lambda-cyhalothrin and this highest observed number of derivative products are not described in the literature, an analytical method by SBSE-GC-IT-MS was developed for application in water control.

\section{Optimization of SBSE extraction and desorption}

The optimization of SBSE extraction using experimental design indicates that the most significant variables in the experimental model are the organic modifier, $\mathrm{pH}$, ionic strength and extraction time. The Pareto chart indicates that the extraction rate of the substances increases as the value of the organic modifier, $\mathrm{pH}$, ionic strength increases and indicates that longer times favor the increase in extraction rates (Figure 1a). The mean effect chart indicates the best rate was achieved with $15 \%$ of methanol, $0.05 \%$ of
$\mathrm{NaCl}, \mathrm{pH} 1.5$ and 120 min of extraction time (Figure 1b). Temperature was not considered to be a significant variable, but the Pareto and mean effect charts demonstrate that a slight increase in extraction occurred at $60{ }^{\circ} \mathrm{C}$ (Figure 1). The position of the central point on the mean effect chart indicates the linearity of the response (Figure 1b). The data interpretation was performed according to the design of experiments described in the literature. ${ }^{22,23}$

The best condition for liquid desorption was determined by performing a kinetic study with the three solvents (methanol, acetonitrile and ethanol). The curve of the desorption kinetics showed a gradual increase in the average desorption rate in response to the increase in sonication time of up to about 90 min with all the tested solvents. After this period of time, the three extraction curves show a logarithmic shape, indicating relevant differences in the average desorption rate of the analyte. Methanol showed the highest average desorption rate $(78.33 \pm 0.17 \%)$, so it was adopted as the desorption solvent for the lambdacyhalothrin derivative products.

In the case of thermal desorption, several temperature conditions (GC injector temperature) were tested and the
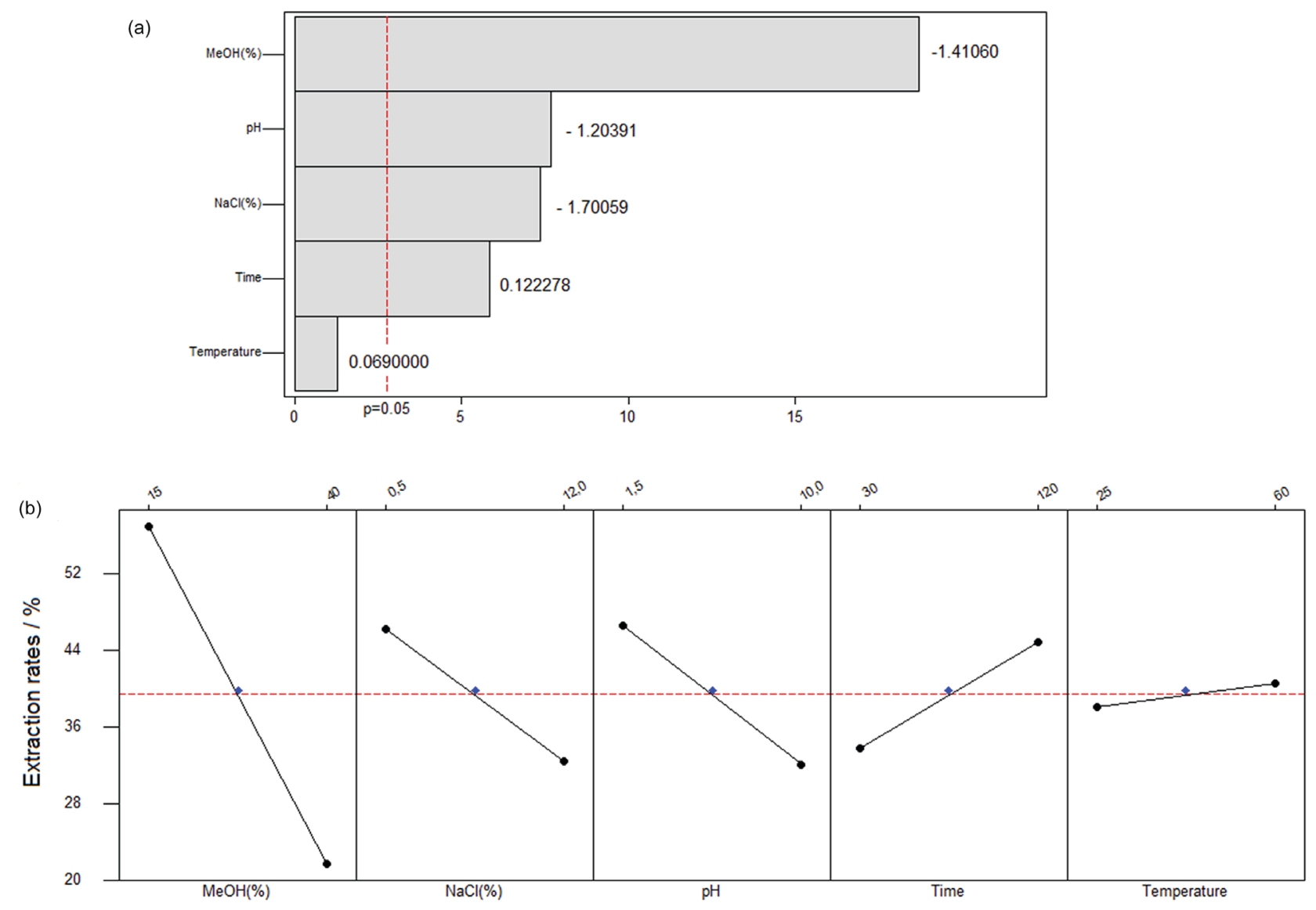

Figure 1. Correlation of the chemical variables on the extraction of lambda-cyhalothrin derivative products using $2^{5-2}$ fractional factorial design: (a) Pareto chart, showing the significant variable in the experimental model ( $\mathrm{MeOH}, \mathrm{pH}, \mathrm{NaCl}$ and extraction time); and (b) mean effect chart, indicating the conditions for the best extraction rate ( $15 \%$ of methanol, $0.05 \%$ of $\mathrm{NaCl}, \mathrm{pH} 1.5$ and 120 min of extraction time). 
optimized desorption conditions were $45{ }^{\circ} \mathrm{C}$ (held for $1 \mathrm{~min}$ ) to $255^{\circ} \mathrm{C}$ (held for $12 \mathrm{~min}$ ), at a heating rate of $42{ }^{\circ} \mathrm{C} \mathrm{min}^{-1}$. Due to the optimization of the SBSE method and the development of the GC methodology for analysis of lambda-cyhalothrin and its derivative products, it was possible to identify the derivative products using SBSE-GC-IT-MS technology.

\section{SBSE-GC-IT-MS identification of the derivative products of lambda-cyhalothrin}

Both SBSE-liquid and SBSE-thermal desorption techniques were effective to analyze the twelve compounds resulting from chemical degradation of lambda-cyhalothrin, as shown in the total ion chromatogram (TIC) profiles (Figure 2).

The twelve proposed compounds (peaks 1-12, Figure 2, Scheme 1 and Table 1) were identified based on the base peak of fragment ions obtained in the full-scan mode and with the support of mass spectral data libraries (NIST MS Search 7.0). The presence of 3-phenoxybenzaldehyde in the reaction solution was also confirmed by comparing the base peak $(\mathrm{m} / \mathrm{z} 198)$ and fragments with those obtained from commercial standards.

An important pathway of the chemical oxidation process involves the cleavage of the carboxyl group of the lambda-cyhalothrin molecule, with retention of the phenoxybenzyl moiety in several compounds remaining after the chemical oxidation process (compounds $\mathbf{2}, \mathbf{3}, \mathbf{5}$, $\mathbf{8}, 10$ and 12). The other derivative pathway of lambdacyhalothrin, also considering the compounds identified by GC-IT-MS data, is cleavage of the ester bond and incorporation of a methylene carbon in the carbon linked to the cyano group.

\section{Conclusions}

This study showed that lambda-cyhalothrin is reactive under simulated environmental conditions, undergoing chemical transformation processes and generating various derivative products. Among them, only 3-phenoxybenzaldehyde is reported as a degradation product of lambda-cyhalothrin, ${ }^{19}$ so the novel data herein shown are relevant for water quality control. This study
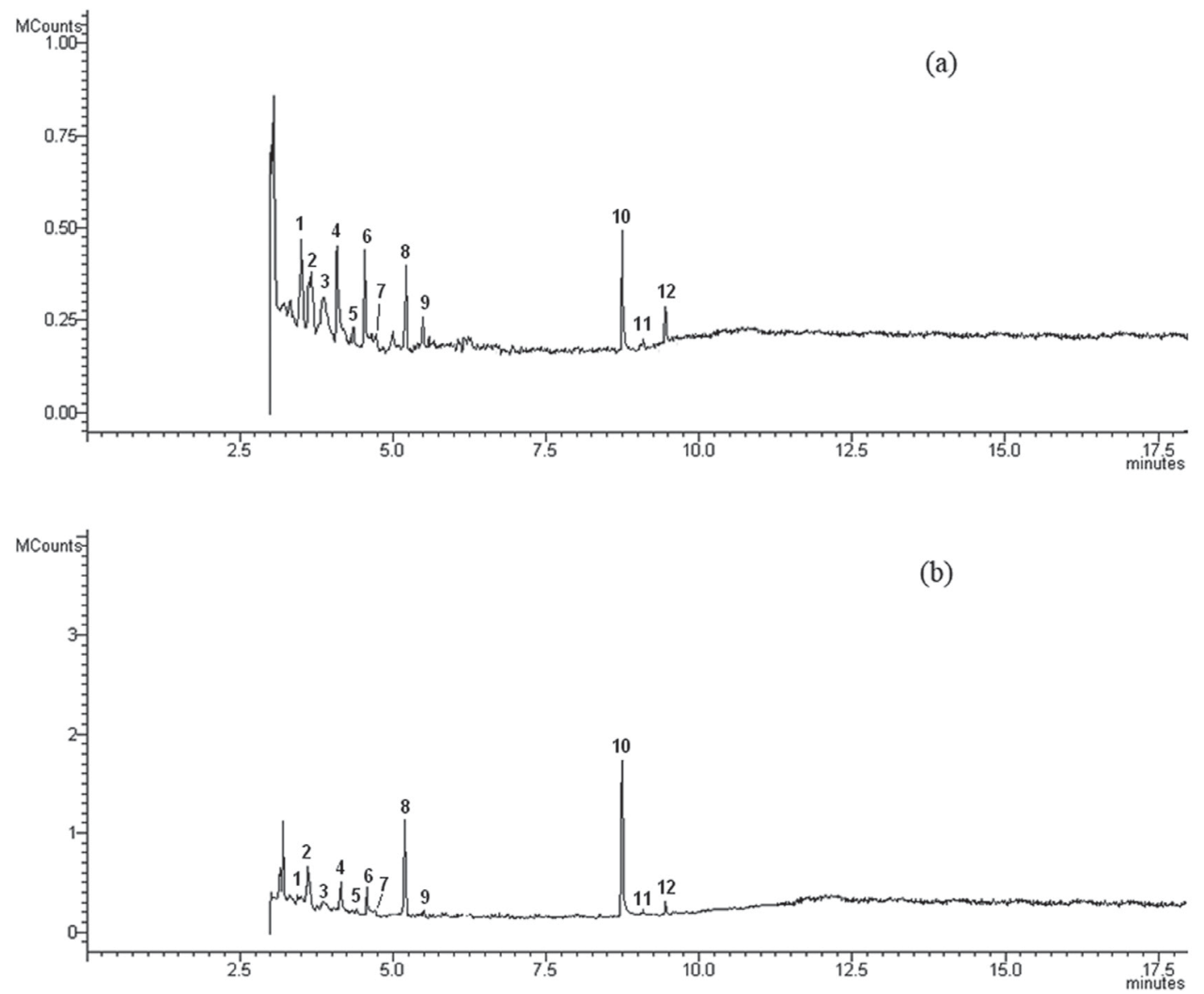

Figure 2. TIC-GC-IT-MS of the derivative products of lambda-cyhalothrin, obtained by (a) SBSE-liquid desorption and (b) SBSE-thermal desorption. The twelve derivative products of lambda-cyhalothrin are effectively desorbed by both SBSE desorption techniques (liquid and thermal). 


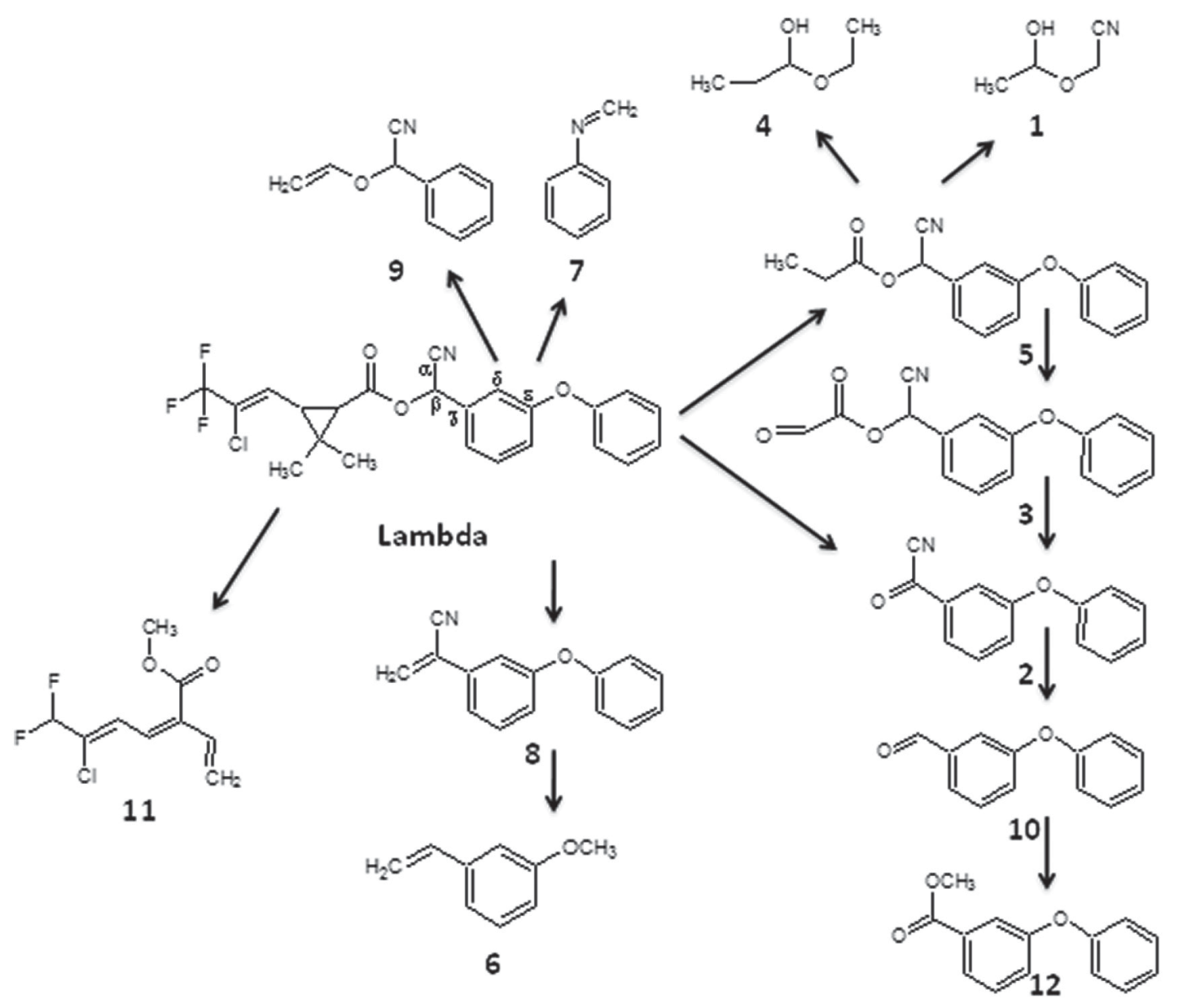

Scheme 1. Proposed derivative pathway of lambda-cyhalothrin by chemical oxidation, based on GC-IT-MS. Data obtained after oxidation at the optimal conditions ( $4.5 \mu \mathrm{g} \mathrm{L}^{-1}$ of lambda-cyhalothrin, $680 \mu \mathrm{g} \mathrm{L}^{-1}$ of hydrogen peroxide, $4 \mathrm{~h}$ of reaction time at $\left.\mathrm{pH} 10.0\right)$. The pathway is coherent with the structure of the twelve identified derivative products of lambda-cyhalothrin, including 3-phenoxybenzaldehyde (10).

Table 1. GC-IT-MS data of the lambda-cyhalothrin derivative products. Peak number as in Figure 2 and Scheme 1

\begin{tabular}{|c|c|c|c|}
\hline Compound & & $\mathbf{M}^{+} / m / z$ & EI-MS fragment ion (relative intensity / \%) \\
\hline 1 & (1-hydroxyethoxy)acetonitrile & 101 & $74(39), 59(36), 44(100)$ \\
\hline 2 & oxo (3-phenoxyphenyl)acetonitrile & 223 & 207 (3), $196(7), 161(29), 141$ (100), 91 (40) \\
\hline 3 & cyano (3-phenoxyphenyl)methyloxoacetate & 281 & $94(100), 66(80)$ \\
\hline 4 & 1-ethoxypropan-1-ol & 104 & $59(3), 44(100)$ \\
\hline 5 & cyano (3-phenoxyphenyl)methylpropanoate & 281 & 253 (44), 223 (40), 209 (16), 115 (26), 44 (100) \\
\hline 6 & 1-ethenyl-3-methoxybenzene & 134 & $102(100), 75(23), 70(70), 59(50), 44(67)$ \\
\hline 7 & $N$-methylideneaniline & 105 (base peak) & 77 (75), $51(35)$ \\
\hline 8 & 2-(3-phenoxyphenyl)prop-2-enenitrile & 221 & 197 (60), $161(53), 141(100), 91(20), 73$ (23) \\
\hline 9 & (ethenyloxy)(phenyl)acetonitrile & 159 & $132(20), 115(100), 69(74), 47(27)$ \\
\hline 10 & 3-phenoxybenzaldehyde & 198 (base peak) & $\begin{array}{c}181(27), 169(93), 141(73), 115(35), 77(26), \\
63(16), 51(52)\end{array}$ \\
\hline 11 & methyl (2Z,4Z)-5-chloro-2-ethenyl-6,6-difluorohexa-2,4-dienoate & 222 (base peak) & 195 (32), 169 (40), $141(30), 115(15)$ \\
\hline 12 & methyl 3-phenoxybenzoate & 228 & 197 (100), 169 (40), 141 (50), 115 (25) \\
\hline
\end{tabular}

EI-MS: electron impact ionization mass spectrometry. 
presents also an effective and innovative method by SBSE-GC-IT-MS, including thermal desorption, an environmentally friendly procedure. This method can be useful to analyze lambda-cyhalothrin and its derivative product in waters from treatment stations or environmental sources

\section{Acknowledgments}

The authors are indebted to FAPESP (grant Nos. 2007/08314-3, 2007/04759-0 and 2013/21886-7) and CNPq (grant Nos. 150772/2010-6, 500200/2010-6 and 302820/2011-6) for the provision of fellowships and financial support.

\section{References}

1. Weston, D. P.; Lydy, M. J.; Environ. Sci. Technol. 2010, 44, 1833.

2. https://www.epa.gov/ingredients-used-pesticide-products/ pyrethrins-and-pyrethroids, accessed in May 2018.

3. He, L. M.; Troiano, J.; Wang, A.; Goh, K.; Rev. Environ. Contam. Toxicol. 2008, 195, 71.

4. Moore, M. T.; Cooper, C. M.; Smith Jr., S.; Cullum, R. F.; Knight, S. S.; Locke, M. A.; Bennett, E. R.; Environ. Pollut. 2009, 157, 250.

5. Clasen, B.; Loro, V. L.; Murussi, C. R.; Tiecher, T. L.; Moraes, B.; Zanella, R.; Sci. Total Environ. 2018, 626, 737.

6. Chang, J.; Hao, W.; Xu, Y.; Xu, P.; Li, W.; Li, J.; Wang, H.; Environ. Pollut. 2018, 232, 309.

7. Wijngaarden, R. P. A. V.; Brock, T. C. M.; Brink, P. J. V. D.; Ecotoxicology 2005, 14, 355.

8. Amweg, E. L.; Weston, D. P.; Environ. Toxicol. Chem. 2005, 24,1300 .
9. Wang, W.; Cai, D. J.; Shan, Z. J.; Chen, W. L.; Poletika, N.; Gao, X. W.; Regul. Toxicol. Pharmacol. 2007, 47, 184.

10. Jiang, W.; Luo, Y.; Conkle, J. L.; Li, J.; Gan, J.; Pest Manage. Sci. 2016, 72, 1411.

11. Zhang, Q.; Zhang, Y.; Du, J.; Zhao, M.; Chemosphere 2017, $185,1173$.

12. Rodrigues, E. T.; Alpendurada, M. F.; Ramos, F.; Pardal, M. A.; Ecotoxicol. Environ. Saf. 2018, 150, 224.

13. https://www.regulations.gov/document?D=EPA-HQOPP-2010-0480-0019, accessed in May 2018.

14. Córdova-Kreylos, A. L.; Lango, J.; Scow, K. K.; Sustainable Agriculture Farming Systems, SAFS Newsletters 2007, 7, 3. Available at http://safs.ucdavis.edu/newsletter/v07n3/safsv07n3.pdf, accessed in May 2018.

15. Feo, M. L.; Eljarrat, E.; Barceló, D.; J. Chromatogr. A 2010 , 1217, 2248.

16. Li, H.; Cheng, F.; Wei, Y.; Lydy, M. J.; You, J.; J. Hazard. Mater. 2017, 324, 258.

17. Aguiar Jr., T. R.; Bortolozo, F. R.; Rosa Filho, E. F.; Parron, L. M.; Luz, L. D.; Brito, A. G.; Ferreira, M. T.; Groundwater Sustainable Dev. 2017, 5, 187.

18. Whitacre, D. M.; Reviews of Environmental Contamination and Toxicology; Springer: Berlin, 2008.

19. https://efsa.onlinelibrary.wiley.com/doi/epdf/10.2903/j. efsa.2014.3677, accessed in May 2018.

20. Minitab, Inc.; Minitab Statistical Software, version 13; Minitab, Inc., State College, PA, USA, 2000.

21. Sherrif, S. S.; Madadi, V.; Int. J. Innov. Res. Sci. Eng. Technol. 2017, 3, 568 .

22. Antony, J.; Design of Experiments for Engineers and Scientists; Elsevier: Oxford, 2003.

23. Anderson, M. J.; Whitcomb, P. J.; DOE Simplified: Practical Tools for Effective Experimentation; CRC Press: New York, 2016. 